www.nature.com/jhg

\title{
Congenital factor XIII deficiency: A commentary on 'Homozygous intronic mutation leading to inefficient transcription combined with a novel frame-shift mutation in F13A1 gene causes FXIII deficiency'
}

\author{
Keiko Shinozawa \\ Journal of Human Genetics (2011) 56, 475-476; doi:10.1038/jhg.2011.53; published online 2 June 2011
}

$\mathrm{T}$

he article entitled 'Homozygous intronic mutation leading to inefficient transcription combined with a novel frameshift mutation in F13A1 gene causes coagulation factor XIII (FXIII) deficiency' by Wang et al. ${ }^{1}$ is interesting. FXIII, which belongs to a family of transglutaminases, is a plasma glycoprotein that has an important role in the stabilization of fibrin clot in the final stage of blood coagulation. FXIII circulates as a heterotetramer, composed of two A (FXIII A) and two B (FXIII B) subunits in plasma. FXIII A possesses catalytic activity, and this catalytic subunit is carried and protected by the FXIII B subunit. Congenital FXIII deficiency is a rare autosomal recessive bleeding disorder affecting one in 1-5 million individuals. People with FXIII deficiency show severe bleeding tendency. The earliest and most characteristic clinical symptom is neonatal umbilical cord bleeding. Other typical findings are impaired wound healing and a high risk of miscarriage. The gene of the FXIII A subunit (F13A1) maps to the short arm of chromosome 6 (p24-25) and spans about $176.75 \mathrm{~kb}$ of genomic DNA. It consists of 15 exons, encoding a mature protein of 732 amino acids. Direct sequence analysis of F13A1 is important in patients with congenital deficiencies and abnormalities of the FXIII A subunit. To date, 111 unique mutations (53 missenses, 19 deletions, 15 splicings, 10 insertions, 10 nonsenses, three gross deletions and

Dr $\mathrm{K}$ Shinozawa is at the Department of Molecular Genetics of Coagulation Disorders, Tokyo Medical University, 6-7-1, Nishishinjuku, Shinjuku-ku, Tokyo 160-0023, Japan.

E-mail: keiko-s@tokyo-med.ac.jp one promoter) have been reported in the FXIII deficiency, and these mutations are described in a database of mutations causing rare bleeding disorders by the International Society on Thrombosis and Haemostasis (Figure 1).

(http://www.isth.org/default/index.cfm/ publications/registries-databases/)

These molecular defects do not appear to be clustered in specific regions and are scattered throughout F13A1. Nevertheless, the majority of missense mutations are located in the catalytic core domain, and no missense mutations have been reported so far in the activation peptide. On the other hand, a splice site mutation in intron 5 (IVS5$1 \mathrm{G}>\mathrm{A})$ seems to be the most common mutation, because it has already been reported in unrelated families from seven different countries.

The article concerned a Chinese patient with congenital FXIII deficiency that was identified by two novel different types of deletion (c.602_605del AAAG) and intronic mutation (IVS1+12C >A), using singlestranded conformation polymorphism analysis and direct sequencing. ${ }^{1}$ The authors found a 4-bp deletion in the patient after electrophoresis of the exon-5 PCR fragment revealed two separate bands. Their subcloning and sequencing revealed a deletion of AAAG at nucleotides 602-605 (c.602_605del AAAG) or AAGA at nucleotides 603-606 in the GAGAAAGAAAGAGAAGAG region, leading to a predicted truncated peptide of 204 amino acid residues. To date, 22 deletions, including three gross deletions, have been described in the F13A1 mutation database, and there are four reports in the database of deletion in exon 5 that is the same as that reported in this article. Duan et al. ${ }^{2}$ reported three unrelated Chinese patients with FXIII deficiency and found three novel mutations, including (c.599del AA). Peyvandi et al. ${ }^{3}$ reported a phenotype-genotype characterization of 10-unrelated Iranian patients and identified a novel small deletion (c.604del A). Similarly, Schroeder et al. ${ }^{4}$ described six novel mutations including (c.607 del G). Although the nucleotide sequences of exon 5 in F13A1 are not strict tandem repeat sequences, the sequence may be predisposed to deletion. In addition, Otaki et al. ${ }^{5}$ identified a gross deletion that is exactly $5984 \mathrm{bp}$ long that includes the entire exon 5.

Another intronic mutation (IVS1+12C $>\mathrm{A}$ ) is of particular interest. There is only one report of a 20-bp deletion in the promoter region, ${ }^{6}$ and no intronic mutation in F13A1 has been reported in FXIII deficiency so far. The first intron of the various kinds of genes in Homo sapiens has been previously suggested to have an expression-augmenting activity. ${ }^{7}$ Previous studies of other genes other than F13A1 have shown that intronic sequences are involved in regulating gene expression. Rohrer and Conley ${ }^{8}$ identified two Sp1 binding sites of intron 1 of the gene for Bruton's tyrosin kinase. Shamsher et al. ${ }^{9}$ identified a regulatory element in intron 1 of the human protein $\mathrm{C}$ gene (PROC). The article by Wang et al. ${ }^{1}$ is the first report in F13A1, although there is a report in other genes like this. To determine the functional effect of the (IVS1+12C $>\mathrm{A}$ ) mutation, the authors found that the first 


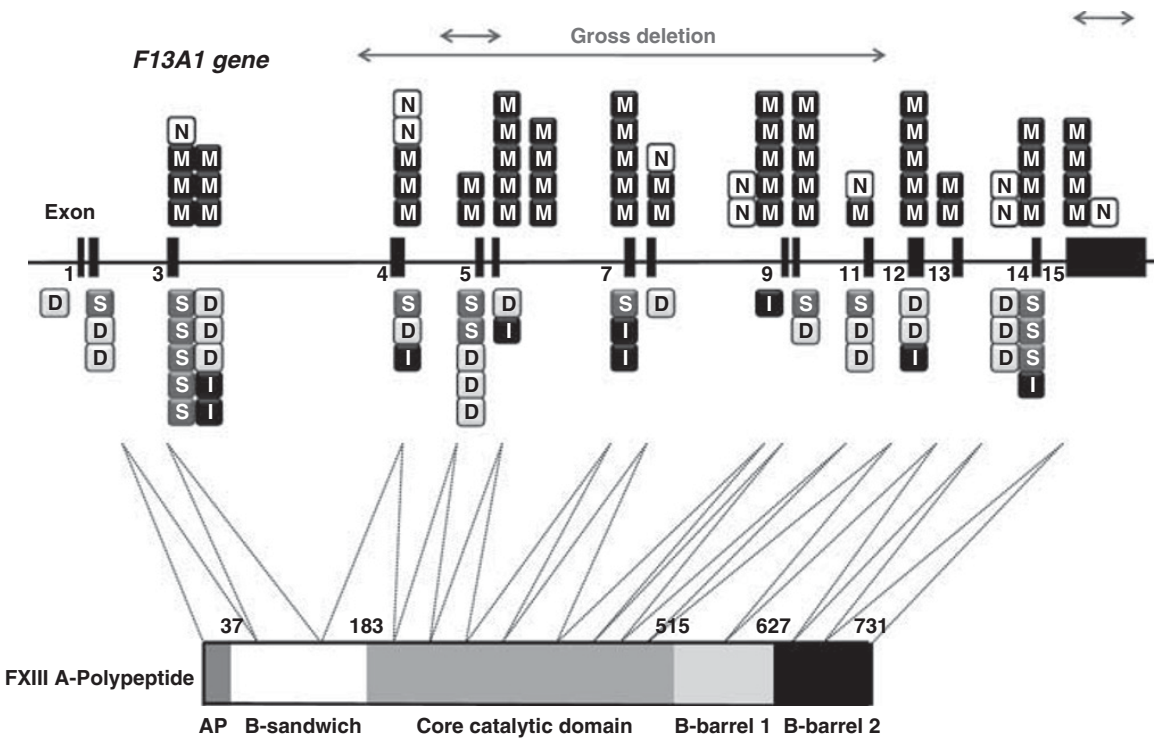

Figure 1 Schematic of factor XIII A subunit (FXIII A) gene (F13A1) indicating the location of mutations of congenital FXIII deficiency and FXIII A-polypeptide. These mutations are described by database of the mutation causing rare bleeding disorders in the International society on thrombosis and Haemostasis (ISTH). (http://www.isth.org/default/index.cfm/publications/registries-databases/) ' $\mathrm{M}$ ' squares indicated missese mutation, ' $\mathrm{N}$ ' squares indicated nonsense mutation, ' $\mathrm{S}$ ' squares indicated splicing mutation, ' $D$ ' squares indicated deletion and ' $\mathrm{l}$ ' squares indicated insertion. Three doubleheaded arrows show gross deletion.

951-bp of intron 1 in F13A1 are involved in regulating the expression of $F 13 A 1$, and that the IVS1+12A mutation results in its reduced expression. Electrophoretic mobility shift assay indicated that the IVS1+12A mutation causes reduced protein binding. An Spl site was predicted in the sequence containing IVS1+12C, which the IVS1+12A mutation eliminates. Co-transfection of a plasmid expressing Sp1 revealed that Sp1 is involved in regulating the expression of FXIII $\mathrm{A}$, and that IVS1+12A leads to inefficient transcription. Whereas the nucleotide of IVS1+12 (NT-007592:g.6260808) is adenine (A) in
ENST0000026480, in fact, the position of this nucleotide is a single nucleotide polymorphism (SNP: rs2815822). The SNP population diversity ratio in Africans and Europeans is approximately 0.9:0.1 (C alleles:A alleles). In Asians, the ratio of minor A alleles is slightly less than 0.05 . In F13A1, initiation codon (ATG) exists in exon 2, and exon 1 is not translated and does not synthesize peptide. The size of intron 1 between exon 1 and exon 2 is $1904 \mathrm{bp}$. It is thought that the intronic mutation that the authors identified is equivalent to a promoter mutation in the $5^{\prime}$-flanking region.
In conclusion, the authors proved that the position of this mutation is a regulatory mechanism in relation to Sp1 binding, although this mutation was classified into polymorphism in a frequency. These results provide the first insight into a novel regulatory mechanism involving intron 1 in the F13A1 gene.

1 Wang, W., Huang, L., Ma, Q., Xiao, D., Chen, X., Yang, Z. et al. Homozygous intronic mutation leading to inefficient transcription combined with a novel frameshift mutation in $F 13 A 1$ gene causes FXIII deficiency. J. Hum. Genet. 56, 460-463 (2011).

2 Duan, B., Wang, X., Chu, H., Hu, Y., Huang, X., Qu, B. et al. Deficiency of factor XIII gene in Chinese: 3 novel mutations. Int. J. Hematol. 78, 251-255 (2003).

3 Peyvandi, F., Tagliabue, L., Menegatti, M., Karimi, M., Komáromi, I., Katona, E. et al. Phenotype-genotype characterization of 10 families with severe a subunit factor XIII deficiency. Hum. Mutat. 23, 98 (2004).

4 Schroeder, V., Meili, E., Cung, T., Schmutz, P. \& Kohler, H. P. Characterization of six novel A-subunit mutations leading to congenital factor XIII deficiency and molecular analysis of the first diagnosed patient with this rare bleeding disorder. Thromb. Haemost. 95, 77-84 (2006).

5 Otaki, M., Inaba, H., Shinozawa, K., Fujita, S., Amano, K. \& Fukutake, K. Characterization of a large deletion that leads to congenital factor XIII deficiency]. Rinsho. Byori. 56, 187-194 (2008).

6 Izumi, T., Nagaoka, U., Saito, T., Takamatsu, J., Saito, H. \& Ichinose, A. Novel deletion and insertion mutations cause splicing defects, leading to severe reduction in mRNA levels of the A subunit in severe factor XIII deficiency. Thromb. Haemost. 79, 479-485 (1998).

7 Kurachi, S., Hitomi, Y., Furukawa, M. \& Kurachi, K. Role of intron 1 in expression of the human factor IX gene. J. Biol. Chem. 270, 5276-5281 (1995).

8 Roher, J. \& Conley, M. E. Transcriptional regulatory elements within the first intron of Bruton's tyrosine kinase. Blood 91, 214-221 (1998).

9 Shamsher, M. K., Chuzhanova, N. A., Friedman, B., Scopes, D. A., Alhaq, A., Millar, D. S. et al. Identification of an intronic regulatory element in the human protein C (PROC) gene. Hum. Genet. 107, 458-465 (2000). 